\title{
A New Fixation Method for the Fracture of Femoral Neck (Delbet Ii) in Children, Case Report
}

\author{
Nan Lü, Yongchuan Li\#, Jiajia Lu, Fan Zhang and Aimin Chen* \\ Department of Orthopaedic surgery, Shanghai Changzheng Hospital, Second Military Medical University, China \\ \#These authors are equally contributed to this work
}

*Corresponding author: Aimin Chen, Department of Orthopaedic surgery, Shanghai Changzheng Hospital, Second Military Medical

University, China

\begin{tabular}{|c|c|}
\hline ARTICLE INFO & ABSTRACT \\
\hline Received: 幽 November 23, 2021 & $\begin{array}{l}\text { Citation: Nan Lu, Yongchuan Li, Jiajia Lu, Fan Zhang, Aimin Chen. A New Fixation Method } \\
\text { for the Fracture of Femoral Neck (Delbet Ii) in Children, Case Report. Biomed J Sci \& Tech }\end{array}$ \\
\hline Published: 豐 January 19, 2021 & Res 33(2)-2021. BJSTR. MS.ID.005369. \\
\hline
\end{tabular}

\section{Introduction}

We report a new fixation method for the fracture of femoral neck in children. For this kind of patients, we got limited methods to achieve reliable stabilization of fracture, because of the existence of osteoepiphysis. K-wires, cannulated screws and locking compression paediatric hip plate were reported in former literatures. Every method has its flaws, we cannot find a perfect method till now.

\section{Case Report}

The patient is a 11-year-old girl. In August 2019, she sustained an injury on her left hip, she fell down from a chair at home. The subsequent X-ray showed a femoral neck fracture in her left hip (Delbet II), totally displaced. She came to our hospital three days after the injury. The X-ray and CT scans confirmed the total displacement of the fracture, no osteoepiphysis involved (Figure 1). In the preoperative discussion, we made the therapy project that we would manage the fracture by internal fixation after reducing the fracture anatomically. Which kind of internal fixation would be adopted remained controversial? K-wires do not have compressive effect on fracture ends. Cannulated screws cannot provide enough stabilization because of the limited length. Locking compression paediatric hip plate may results in longer operation time and more blood loss compared with cannulated screws and K-wires. So, we decided to adopt both K-wires and cannulated screws, in order to avoid the defects. After necessary preoperative preparation, we proceeded with reconstructive surgery. Under general anesthesia, we first made close reduction with the use of traction table and got a mostly satisfactory result. Then we put a K-wire into the femoral head and achieved anatomical reduction by stirring the K-wire.
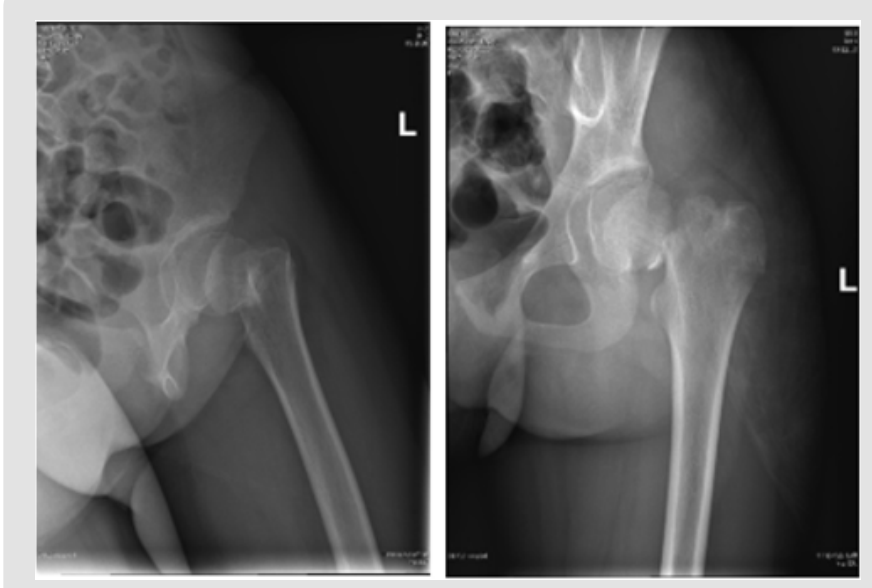

Figure 1: Preoprative $X$-rays.

Three $0.5 \mathrm{~cm}$ incision was made in the lateral side, three $\mathrm{K}$-wires were put into the femoral neck under monitoring by the C-arm fluoroscopy. Every K-wire's end was 2-3 mm under the osteoepiphysis. Three cannulated screws were screwed into the femoral neck and the fracture was compressed. Three K-wires were further knocked into the femoral neck with a hammer. Every 
K-wire's end was 2-3 mm under the cartilage of femoral head. Then the other ends of K-wires were bended and cut off. After washed with normal saline, the incision was stitched (Figure 2).
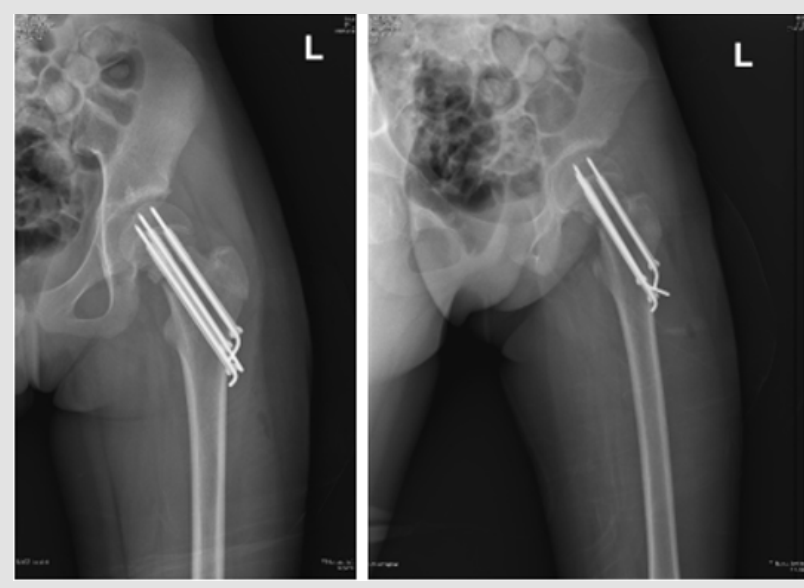

Figure 2: Postoperative X-rays.

\section{Discussion}

Fractures of femoral neck in children are uncommon [1-4], early surgical treatment is widely advised [5,6]. Femoral neck fractures of pediatric age were initially classified according to the four-part classification system described by Delbet and popularized by Colonna [6]. Type I is an injury of the proximal femoral physis. Type II is a transcervical fracture. Type III is cervicotrochanteric fracture. And type IV is an intertrochanteric fracture. The first three types are intra-articular fractures, and the last type is extracapsular fracture. Delbet type II is the most common fracture according to previous literatures [3,7]. The existence of epiphyseal cartilage might restrict blood supply to femoral head from femoral neck in children [8]. Femoral neck fracture in children which caused by high energy injury might result in more avascular necrosis of femoral heads than adults [9]. Anatomical reduction and compressed fixation of femoral neck fracture could reduce the damage to the blood supply of femoral head. The choice of implants depends on several factors. Such as operating surgeon's preference, the patient's age, and size et'al. Several methods were reported in former literatures, such as K-wires, cannulated screws and locking compression paediatric hip plate. Every method has its own advantages and disadvantages. K-wires do less harm to epiphysis, while have no compressed effect. Long cannulated screws can't be applied because the screw threads may do harm to epiphysis. How could we avoid the disadvantages while retain the advantages of both these two methods?
K-wires were used as guide needle during usual operations. The cannulated screws cannot be put through the epiphysis, while $\mathrm{k}$-wires can. In this operation, k-wires were first used as guide needle, they were knocked deeper before the hollow drill was applied. Hammer instead of electric drill was used to knocked $\mathrm{k}$-wires for rapidly rotating k-wires might do harm to epiphysis. After the cannulated screws were put, intraoperative fluoroscopy was used in avoid of hip joint cavity invasion of K-wires. Then K-wires tail bending were applied in avoid of hip joint cavity invasion. We got more stable fixation by long enough $\mathrm{K}$-wires, and compressive effect by cannulated screws.

\section{Conclusion}

The fractures of femoral neck (Delbet II) in children are not common. The combination of K-wires and cannulated screws are reliable for the fracture. They are complementary methods.

\section{Conflict of Interest}

All authors declare that they have no conflict of interest.

\section{Acknowledgement}

None.

The patient gave informed consent, and the Ethical Committee of the Second Military Medical University approved this study.

\section{References}

1. Ratliff $A H$ (1962) Fractures of the neck of the femur in children. J Bone Joint Surg Br 44: 528-542.

2. Canale ST, Bourland WL (1977) Fracture of the neck and intertrochanteric region of the femur in children. J Bone Joint Surg Am 59: 431-443.

3. Togrul E, Bayram H, Gulsen M, Kalaci A, Ozbarlas S (2005) Fractures of the femoral neck in children: Long-term follow-up in 62 hip fractures. Injury 36: 123-130.

4. Ratliff AH (1974) Fractures of the neck of the femur in children. Orthop Clin North Am 5: 903-921.

5. Swiontkowski MF, Winquist RA (1986) Displaced hip fractures in children and adolescents. J Trauma 26: 384-388.

6. Smith LD (1953) Hip fractures: The role of muscle contraction or intrinsic forces in the causation of fractures of the femoral neck. J Bone Joint Surg Am 35a(2): 367-383.

7. Bali K, Sudesh P, Patel S, Kumar V, Saini U, et al. (2011) Pediatric femoral neck fractures: Our 10 years of experience. Clin Orthop Surg 3: 302-308.

8. Hughes LO, Beaty JH (1994) Fractures of the head and neck of the femur in children. J Bone Joint Surg(Am) 76(2): 283-292.

9. Vito Pavone, Gianluca Testa, Maria Riccioli (2019) Surgical treatment with cannulated screws for pediatric femoral neck fractures: A case series. Injury Int J Care Injured 2: S40-S44. 
ISSN: 2574-1241

DOI: 10.26717/BJSTR.2021.33.005369

Aimin Chen. Biomed J Sci \& Tech Res

(C) This work is licensed under Creative

Submission Link: https://biomedres.us/submit-manuscript.php

\begin{tabular}{ll} 
BIOMEDICAL & Assets of Publishing with us \\
RESEARCHES & - Global archiving of articles \\
- Immediate, unrestricted online access & - Rigorous Peer Review Process \\
\hline
\end{tabular}

